\title{
Cuidados y sostenibilidad de la vida: Una reflexión a partir de las políticas de tiempo
}

\section{Papeles del CEIC ISSN: $1695-6494$}

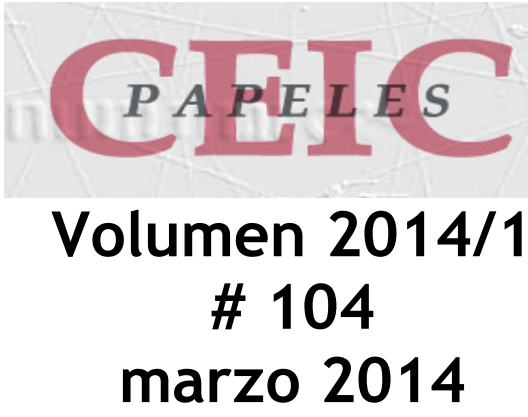

\section{Resumen}

Cuidados y sostenibilidad de la vida: Una reflexión a partir de las políticas de tiempo

\section{Matxalen Legarreta Iza}

Dpto. Sociología 2

Universidad del País Vasco - Euskal Herriko Unibertsitatea

E-mail: matxalen.legarreta@ehu.es

Las políticas de tiempo surgen en Italia a finales de 1980

a través de una propuesta de ley de iniciativa popular Time policies emerge in Italy in late 1980 through a que fija las bases de un campo de investigación e popular initiative. Such iniciative provides the basis of a intervención cuyo potencial ha sido explorado solo en research and intervention field which explores its parte. El texto tiene como objetivo llevar a cabo una potential only partially. The text aims to carry out a rerelectura de dicha iniciativa en torno a tres aspectos reading of the initiative around three core issues of the medulares de los debates más recientes y novedosos recent debates on care and sustainability of life: social sobre cuidados y sostenibilidad de la vida: desigualdades inequalities and processes of empowerment; autonomy, sociales y procesos de empoderamiento; autonomía, dependency, vulnerability; and social structuring and dependencia, vulnerabilidad; y estructuración y cambio change. The reflection lands on a concrete example: social. La reflexión refleja en un ejemplo concreto: los time banks. bancos de tiempo.

Palabras clave

Políticas de tiempo, cuidados, sostenibilidad de la vida, bancos de tiempo

\section{Key words}

Time policies, care, sustainability of life, time banks.

\section{Índice}

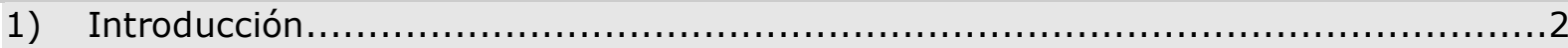

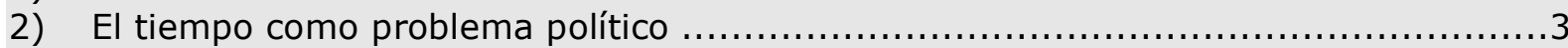

3) Desigualdades, relaciones de poder y procesos de empoderamiento $\ldots \ldots \ldots \ldots \ldots \ldots \ldots . . . \ldots$

4) Autonomía, dependencia y vulnerabilidad........................................... 14

5) Más allá de la conciliación: hacia una nueva organización social .....................19

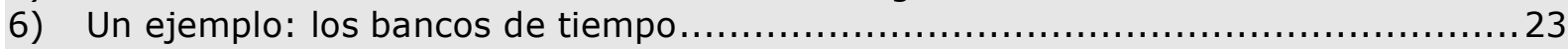

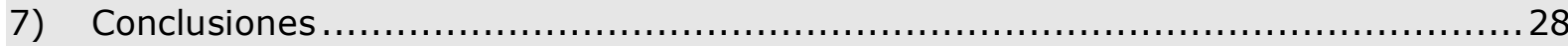

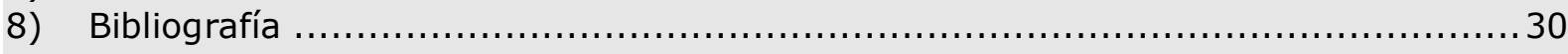




\section{1) INTRODUCCIÓN}

Las políticas de tiempo nacen en Italia a finales de 1980 a partir de una propuesta de ley desarrollada por las mujeres del entonces Partido Comunista. La iniciativa marca un hito importante en este terreno, sin embargo, las experiencias que se han implementado posteriormente, tanto en Italia como en el resto de Europa, se caracterizan por su trayectoria relativamente corta, fragmentaria y desigual, así como por no llegar a desarrollar el potencial que encierra la propuesta inicial. Por ello, resulta pertinente volver a sus orígenes y recuperar la propuesta de ley italiana, para ofrecer una lectura renovada de la misma.

Con dicha finalidad, en este artículo se pretende llevar a cabo una revisión de la propuesta de ley en el marco de los debates más actuales y novedosos en torno a los cuidados y la sostenibilidad de la vida. Tal revisión se elabora en un doble sentido. Por un lado, se pretende realizar una contribución en torno a las políticas de tiempo sacando a la luz el potencial y la relevancia actual de la propuesta de ley. Y, por otro, se aspira a realizar una aportación en torno a los debates sobre los cuidados y la sostenibilidad de la vida mostrando el potencial del tiempo como herramienta analítica. De esta forma, se abordan las políticas de tiempo desde la centralidad de los cuidados y la satisfacción de las necesidades humanas a partir de una mirada situada, sensible a las dinámicas del día a día y, paralelamente, atenta a los procesos que acontecen a nivel estructural. Asimismo, el tiempo sirve en este caso de instrumento para llevar a cabo una reflexión sobre la vida social, así como para sacar a la luz las ocupaciones cotidianas desempeñadas tradicionalmente por las mujeres en los entornos doméstico-familiares, dar cuenta de sus características particulares, dotarlas de valor analítico y favorecer su reconocimiento social.

El artículo se divide en cinco grandes apartados, además de la introducción y las conclusiones. El primero, ofrece un acercamiento a la 
propuesta de ley italiana, da cuenta de sus fundamentos principales y presenta el marco interpretativo en el que se va a analizar en las siguientes secciones. Los siguientes tres epígrafes se desarrollan con el propósito de tratar la propuesta de ley en relación a algunas de las cuestiones clave de los debates sobre los cuidados y la sostenibilidad de de vida: desigualdades y procesos de empoderamiento; autonomía, dependencia y vulnerabilidad; estructuración y cambio social. El segundo, aborda las desigualdades de género y otro tipo de desigualdades sociales que devienen de la centralidad que toma el ámbito productivo-mercantil en la definición y estructuración contemporánea del tiempo, así como los procesos de captación y el poder de decisión sobre el propio tiempo. El tercero, plantea la reflexión en torno a la dicotomía autonomía/dependencia y el desarrollo de una noción universal, extensa e inclusiva de vulnerabilidad. El cuarto, se centra en la estructuración y el cambio social para dar cuenta de las formas en las que se organiza la vida social a nivel macro. El quinto, refleja las argumentaciones previas en un ejemplo concreto, los bancos de tiempo, para poner de manifiesto tanto sus puntos fuertes como sus limitaciones. Por último, se presentan las conclusiones.

\section{2) El tiempo COMO PROBLema político}

El tiempo se define como problema político en Italia a partir de la propuesta de ley de iniciativa popular que lleva por título Le donne cambiano il tempo (Las mujeres cambimos el tiempo). La iniciativa emerge del diálogo entre la academia y el movimiento feminista y tiene como objetivo último incentivar un proceso de debate sobre los fundamentos en los que se basa la estructura social, para convertirse en una "hipótesis de cambio" de la manera de entender el tiempo, el trabajo y las funciones de mujeres y hombres en la sociedad (Cordoni, 1993: 223). La propuesta se termina de redactar el 4 de abril de 1990 y en octubre del mismo año se presentan 300.000 rubricas en el 
Congreso ${ }^{1}$. Pese a que no llega a constituirse como ley en su totalidad, sirve para impulsar normativas y experiencias interesantes tanto en Italia como en el conjunto de Europa. Cabe afirmar, por tanto, que en el momento en el que surge logra un eco importante en la sociedad italiana y, a posteriori, proporciona los fundamentos de una línea de investigación e intervención desarrollada en Europa en torno a las políticas de tiempo.

Concretamente, la iniciativa nace con el objetivo de avanzar hacia una distribución más equitativa del tiempo, que supere el esquema tradicional de jerarquización establecido por la división sexual del trabajo en torno a las dicotomías producción/reproducción, público/privado, masculino/femenino, y que tome en consideración las distintas necesidades de mujeres y hombres a lo largo de todo el ciclo vital. Con dicha pretensión, promueve una intervención trasversal que atraviesa diferentes aspectos de la vida cotidiana, cuestionando la centralidad del tiempo del mercado como eje estructurador. De este modo, se reconoce el tiempo como problema político: se problematiza la definición hegemónica del tiempo, así como la configuración social de la ordenación temporal vigente, poniendo de manifiesto su carácter social, histórico y contextual, y las relaciones de poder sobre las que descansa. El tiempo se concibe, por tanto, como una construcción social sobre la que se puede intervenir para avanzar hacia el cambio social. La propuesta se concreta en relación a tres ámbitos: el ciclo vital, el tiempo de trabajo y el tiempo de la ciudad.

Sobre los tres pilares mencionados y tomando el tiempo como herramienta, la iniciativa desarrolla una crítica integral de la estructuración

\footnotetext{
${ }^{1}$ Se pueden encontrar textos traducidos al castellano sobre los pilares de la propuesta de ley, el marco en el que surge y las iniciativas concretas que plantea en el número 42 de la Revista Mientras Tanto (1990) (Grau, 1990; Sección femenina nacional del PCl, 1989/1990; Balbo, 1990; Pennacchi, 1990). El artículo de Elena Cordoni (1993) ofrece también un desarrollo pormenorizado de dichos aspectos. Asimismo, el libro editado por Laura Balbo (1991) recoge los fundamentos de los debates conceptuales en torno al trabajo y el tiempo que se desarrollan en el contexto de la elaboración de la propuesta de ley.
} 
social y del lugar que ocupan en ella las mujeres y los hombres. Los fundamentos en los que se concreta se dividen en tres apartados que engloban un total de treinta y dos artículos. Cada apartado hace referencia a un ámbito de intervención concreto que se corresponde con una dimensión temporal: el tiempo en el arco de la vida, el tiempo en el trabajo y el tiempo en la ciudad. Siguiendo el trabajo de Ramón Ramos en torno a las metáforas de tiempo en la vida cotidiana (Ramos, 2005, 2007, 2009, 2011), se puede constatar que en la iniciativa se abordan desde los aspectos temporales más íntimos y personales (tiempo incorporado, encarnado), hasta los externos y estructurales (tiempo como escenario o entorno), pasando por un nivel intermedio en el que el tiempo se concibe como un recurso del que eventualmente se dispone para poder obrar. De este modo, se apela a tres dimensiones del tiempo que son diferentes entre sí, a la par que complementarias. Cabe afirmar, por tanto, que en la propuesta se emplea una noción de tiempo que responde a los postulados de la epistemología feminista: plural, multidimensional, nojerárquico y unido a la experiencia (Adam, 1990; Leccardi, 1996). De ahí deriva uno de sus puntos fuertes.

Respecto al primer apartado, el tiempo en el arco de la vida, la propuesta cuestiona la linealidad del modelo de desarrollo vital articulado a partir del tiempo dedicado al mercado laboral. El tiempo de mercado es el que estructura la trayectoria de vida del modelo masculino hombre ganador de pan que se erige como patrón universal. A la hora de elaborar la reflexión, la iniciativa italiana parte de una mirada diacrónica que permite observar que el modelo de ciclo vital hegemónico se configura según una ordenación temporal lineal compuesta por una secuencia (previsible) en la que se diferencian tres etapas: niñez y juventud como periodo para la formación (preparación para el mercado), edad adulta como momento para la participación en el mercado, y vejez como etapa para la jubilación (retirada del mercado). La propuesta de ley apunta que una ordenación tal no contempla el tiempo que es preciso dedicar 
al trabajo doméstico y a los cuidados, ni la importancia de los ritmos biológicos en el desarrollo vital (como por ejemplo, la edad reproductiva de las mujeres). Una concepción lineal del ciclo vital omite, asimismo, la intermitencia de las experiencias femeninas en relación al mercado, que no se caracterizan por la continuidad y la sucesión, sino por la discontinuidad (entradas y salidas), la simultaneidad, y el encaje temporal entre los tiempos laborales y otros tiempos (los de cuidado, principalmente).

El enfoque relacionado con el ciclo vital ha sido el que menor desarrollo ha tenido en Europa (Torns, Borrás, Moreno y Recio, 2008): se implementa en torno a programas como Lifelong Learning de la Comisión Europea o en relación a políticas de envejecimiento activo (Del Moral, 2012). Cabe advertir, además, que dichas iniciativas no se autodefinen como políticas de tiempo.

En referencia al segundo apartado, el tiempo en el trabajo, la propuesta italiana pone en tela de juicio la linealidad de la ordenación temporal de la vida cotidiana fundamentada sobre la centralidad del trabajo retribuido. Para ello, parte de una mirada sincrónica que ofrece la posibilidad de observar que, reemplazando los ritmos circadianos, la estructuración lineal de lo cotidiano divide el día de forma secuencial y predecible en tres momentos sucesivos: ocho horas de trabajo, ocho horas de tiempo libre y ocho horas de descanso. Dicha estructuración oculta la existencia misma de otros tiempos no tan fácilmente programables ni previsibles: tiempos dedicados a la reproducción de la vida, a cubrir las demandas y necesidades del ámbito doméstico-familiar. La iniciativa saca a la luz la necesaria existencia de un tiempo de cuidado que, en el caso de las mujeres, se matiza, no debe ser definido como tiempo libre pues, como posteriormente se subraya también en otros trabajos (Torns, 2007; Esquivel, 2009; Molinier, 2011, entre otras), se apela a que el cuidado es ante todo trabajo. 
Las políticas de tiempo de trabajo cuentan con una trayectoria más dilatada que las políticas relacionadas con el ciclo vital. Se concretan en normativas y medidas implementadas tanto a escala nacional (la semana de 35 horas en Francia, el modelo Finlandés 6+6 y las medidas Work \& Life Balance en el Reino Unido, por ejemplo) como a nivel europeo (políticas de conciliación). No obstante, no han tenido el éxito esperado porque se sigue primando una cultura del trabajo presencial y una reducción de la jornada laboral diacrónica y acumulativa, frente a la disminución sincrónica y cotidiana de horas (Torns, Borrás, Moreno y Recio, 2008).

En cuanto al tercer apartado, el tiempo en la ciudad, la propuesta italiana constata una desincronización entre los ritmos colectivos e individuales de la vida cotidiana que afecta principalmente a las mujeres. Apunta que dicha situación deriva de la falta de adecuación de los horarios de los diversos servicios y la falta de correspondencia entre éstos y los horarios laborales, así como de la escasa coordinación entre los diversos agentes que participan en la toma de decisiones en torno a los horarios de los servicios públicos y privados. Según se afirma, tal desencaje afecta de forma más acusada a las mujeres ya que son ellas las que, al asumir tradicionalmente las responsabilidades doméstico-familiares, se encargan de la mediación entre las demandas y necesidades de los miembros de la familia y los servicios públicos y privados que las cubren. La iniciativa pone de manifiesto que la vigente forma de entender la organización temporal de la ciudad asume la división de funciones, tareas y responsabilidades del modelo hombre ganador de pan-mujer ama de casa $^{2}$. Así, naturaliza tanto el papel de las mujeres como mediadoras entre la

\footnotetext{
${ }^{2}$ Hoy en día el modelo hombre ganador de pan-mujer ama de casa no se corresponde de forma mimética con las prácticas cotidianas de las sociedades occidentales contemporáneas. Jane Lewis (2007/2011) señala una tendencia de cambio un modelo de "un trabajador y medio y una sola cuidadora" (participación parcial de las mujeres en el mercado laboral), hacia la figura de "dos trabajadores y una sola cuidadora" (una participación generalizada de ellas en el mercado laboral, no correspondida con un protagonismo similar por parte de ellos en el entorno doméstico-familiar). Pese a ello, el ideal sigue vigente en el imaginario social (Legarreta,
} 2013a). 
familia y los servicios, como la asunción por parte de ellas de las ocupaciones, deberes y responsabilidades del entorno doméstico-familiar; al tiempo que no toma en consideración que, cada vez en mayor medida, ellas participan también en el mercado laboral.

Las políticas de tiempo de la ciudad se han desarrollado con cierto éxito en Europa, siendo Italia el país pionero. No se crean en condiciones similares, ni se desenvuelven en la misma dirección, por lo que contemplan diversas y diferentes medidas de actuación. En Alemania, por ejemplo, se promueven principalmente desde la sociedad civil, mientras que en Francia se apoyan en mayor medida en las decisiones políticas (Mückenberger, 2007). En nuestro contexto más cercano cabe mencionar la instauración de una Concejalía de Nuevos Usos Sociales del Tiempo (NUST) en Barcelona a finales de $2003{ }^{3}$ que se desarrolla principalmente en torno a las políticas de tiempo y de trabajo (Legarreta, 2010).

$\mathrm{Si}$, a partir de los ejemplos mencionados, es posible afirmar que las políticas de tiempo han tenido cierto calado en Europa, se ha de advertir que cuentan con un desarrollo parcial y desigual: no existen experiencias que cumplan de forma integral con los objetivos y las demandas de la iniciativa italiana, ni todas contemplan la perspectiva de género $y$, a menudo, se identifican como políticas de tiempo únicamente las relacionadas con los tiempos de la ciudad ${ }^{4}$. Se puede afirmar, por tanto, que la propuesta de ley italiana encierra un potencial que, hoy por hoy, no ha sido suficientemente explorado.

\footnotetext{
3 Actualmente la Concejalía sigue vigente como línea de trabajo del Ayuntamiento de Barcelona. Para más información se puede consultar su página web: http://w110.bcn.cat/portal/site/UsosDelTemps?lang=es ES (Consultado el 07/01/2014).

${ }^{4}$ Se realiza un análisis del desarrollo y alcance de las políticas de tiempo en Europa en: Boccia (2003), Bonfiglioli (2008 y 2009), Boulin y Mückenberger (1999), Boulin (2006), Del Moral (2012), Mückenberger (2007, 2008, 2011), Lombardo y Sangiuliano (2008), Recio (2009) y Torns, Borrás, Moreno y Recío (2008), entre otros. 
En los siguientes epígrafes se analiza la propuesta de ley en el marco de los debates sobre los cuidados y la sostenibilidad de la vida. Para ello, se hace referencia tanto a los fundamentos y a las medidas concretas planteadas en su surgimiento como, de forma más breve, a su desarrollo posterior en relación a las políticas de tiempo. Cabe señalar que el objetivo principal es subrayar el potencial de la propuesta de ley, y no tanto llevar a cabo una revisión exhaustiva de su alcance ni de su desarrollo efectivo. Asimismo, se explora la posibilidad que ofrece la iniciativa italiana para abordar el potencial del tiempo en tanto que herramienta analítica, concretamente, ahondando en la dimensión política del tiempo (Legarreta, 2013b).

\section{3) Desigualdades, Relaciones De poder y Procesos de EMPODERAMIENTO}

El primer eje de los debates actuales sobre los cuidados y la sostenibilidad de la vida que resulta pertinente profundizar en relación a la propuesta de ley italiana y a las políticas de tiempo es el relacionado con las desigualdades, las relaciones de poder y los procesos de empoderamiento.

Poniendo el foco de atención en los procesos de negociación y el poder de decisión sobre el propio tiempo, el tiempo sirve como instrumento para sacar a la luz relaciones de poder asimétricas y dar cuenta tanto de las desigualdades sociales estructurales, como de situaciones específicas de subordinación y de abuso. Una mirada desde el tiempo ofrece, asimismo, la posibilidad de desarrollar un acercamiento dinámico y multidireccional a las relaciones de poder $^{5}$, al tiempo que permite dar cuenta del aumento de la

${ }^{5}$ Desde una perspectiva de tiempo-entorno, se observa que la distribución del trabajo doméstico y los cuidados, al igual que la responsabilidad en torno a ellos, cambian según el momento vital (mirada diacrónica) y la posición y el estatus dentro de la familia (mirada sincrónica); mientras que, desde una perspectiva de tiempo-recurso se puede dar cuenta de las estrategias de apropiación de tiempo (Legarreta, 2013c). 
autonomía y de la capacidad de decisión de las mujeres en relación a los procesos de individuación y desinstitucionalización (Beck y Beck-Gersteim, 2001a, 2001b).

Partiendo de la crítica al modelo hombre ganador de pan-mujer ama de casa así como del cuestionamiento de la centralidad del mercado en la estructuración de la vida social, del día a día y del ciclo vital, la propuesta italiana desarrolla un diagnóstico que pone de manifiesto la persistencia de las desigualdades de género, así como de otro tipo de desigualdades sociales. Paralelamente, la iniciativa propone varias estrategias para hacer frente a dichas desigualdades que, en parte, se han implementado en el marco de las políticas de tiempo de la ciudad: revalorización del trabajo doméstico y los cuidados, poder de decisión sobre el tiempo propio y desarrollo de mecanismos para activar la participación social.

En relación a la revalorización del trabajo doméstico y los cuidados, así como del tiempo que es preciso emplear en ellos, la iniciativa italiana proclama el valor a todos los trabajos para avanzar hacia un reparto equitativo tanto de las ocupaciones remuneradas como de las no retribuidas. Con tal finalidad, se plantea el desarrollo de un tiempo de vida: un tiempo que no responde a los requerimientos del mercado, sino que está atento a las necesidades de las personas. En este contexto, se apuesta por "afirmar la dignidad de todos los tiempos y revalorar la vida cotidiana, modificando actitudes, comportamientos y relaciones de poder entre hombres y mujeres" (Cordoni, 1993: 225). Se puede constatar, por tanto, que a partir de la noción de tiempo de vida, se sitúa en el centro del debate político y social la sostenibilidad de la vida, con el objetivo de trastocar la división de roles, de tareas, de responsabilidades y de tiempos, al igual que su jerarquización.

Desde esta mirada, en tanto que se subraya el valor social del tiempo de cuidados, se aboga por una relación bidireccional en torno a ellos: garantizar 
que todas las personas tengan la posibilidad tanto de recibirlos como de prestarlos, en cualquier momento del ciclo vital. Un posicionamiento tal conlleva varias implicaciones. Por un lado, se propone hacer efectivo el derecho y la capacidad de todas las personas de dar y de recibir cuidados, dotando de voz propia a la población más frágil (criaturas, población anciana, y otras personas que precisan especial atención) por considerar que, hasta el momento, "han sido representadas por medio de las mujeres" (Cordoni, 1993: 225). De este modo, siguiendo otras propuestas, la iniciativa reivindica la agencia de las "identidades quebradas, desgarradas" (Garro, en prensa), para dotarlas de valor y reconocimiento. Por otro lado, las mujeres dejan de ser sujetos dependientes y sale a la luz su agencia como generadoras de tiempo (Leccardi, 1996; Davies, 1990) a la hora de procurar la subsistencia y el bienestar de los demás miembros del hogar como, por ejemplo, en tanto que mediadoras entre las instituciones y los servicios públicos y las demandas de los miembros del entorno doméstico-familiar.

En relación a la proclamación de la autogestión del tiempo propio, la propuesta italiana afirma el derecho de todas las personas de hacer uso de la capacidad de decisión en torno a su propio tiempo. De tal modo, se pone en tela de juicio "el modelo de vida a tiempo único" del ideal hombre ganador de pan-mujer ama de casa: regido para ellos principalmente por las demandas y necesidades del mercado y para ellas por las exigencias del ámbito domésticofamiliar. La autogestión del tiempo propio promueve el poder de decisión, la capacidad de acción y el empoderamiento de todas las personas. Desde esta mirada, se apela a una noción de tiempo en tanto que recurso político, desarrollada en otros trabajos (Ramos, 2007, Legarreta, 2013c), pues lo que está en juego es la capacidad de acción de las personas, su agencia. Para hacer efectiva esta dimensión, la iniciativa italiana propone varias medidas, entre las que se puede destacar la creación de comisiones de consulta permanente sobre tiempo con un protagonismo especial por parte de las 
mujeres (en tanto que siguen asumiendo su tradicional función de mediación entre las familias y los servicios), así como el desarrollo de planes de ordenación de los tiempos que otorguen a los Ayuntamientos el poder de ordenar y coordinar los horarios que regulan las actividades y el ritmo de la ciudad.

Este tipo de iniciativas se han implementado en Italia con cierto éxito, siendo pionera la ciudad de Módena (Recio, 2009). Así, en 1991 se aprueba en el país la ley 142/90 que conlleva una reforma de las autonomías locales y que reconoce a los alcaldes y las alcaldesas la posibilidad de administrar de forma autónoma los horarios públicos y privados de su ciudad según las exigencias de la ciudadanía; Florencia, Milán, Roma y Turín activan este tipo de planes (Boccia, 2003; Bonfiglioli, 2008). Y en el año 2000, con la aprobación de la conocida como Ley Turco, se instituyen las oficinas de tiempo en ciudades de más de 30.000 habitantes y se fijan las bases del desarrollo de las políticas de tiempo en todo el país a partir de la implementación de leyes regionales, principalmente en zonas del centro-norte (Bonfiglioli, 2008) ${ }^{6}$.

En relación al desarrollo de mecanismos para activar la participación social, es posible afirmar que parte de las políticas de tiempo de la ciudad nacen con el objetivo de impulsar el fortalecimiento de lazos sociales y de desarrollar procesos que promuevan un aumento de la capacidad de participación de la ciudadanía. Como se señala en otros trabajos, en su posterior desarrollo, las políticas de tiempo de la ciudad se constituyen como políticas de participación que activan procesos de debate, de negociación y de acuerdo entre las necesidades personales y las exigencias colectivas, y actúan transversalmente en varias esferas de la vida cotidiana, entremezclando

\footnotetext{
${ }^{6}$ El 8 de Marzo de 2000 se aprueba la Ley 53/00 (conocida como Ley Turco) que regula tres aspectos fundamentales de la vida cotidiana incluidos por primera vez en una misma normativa: bajas por maternidad y paternidad, bajas por derechos al cuidado y a la formación, y coordinación de los horarios dentro de las ciudades. Se realiza una reflexión sobre la implementación y el desarrollo de la Ley en Lombardo y Sangiuliano (2008).

Matxalen Legarreta 
distintos ámbitos y diferentes formas de declinar las vivencias de los diversos grupos sociales (Boccia, 2003). Se dirigen hacia la creación y consolidación de tiempos colectivos y de sociabilidad $y$, tanto por su carácter trasversal como por la relevancia que otorgan al diálogo social, se ha llegado a afirmar que pueden convertirse en elementos clave para avanzar hacia una "nueva forma de gobernabilidad" (Boulin, 2006: 201) y plantear "un nuevo reto para el Estado de Bienestar" (Torns, 2004).

En este sentido, se apunta también el potencial de las políticas de tiempo de la ciudad para el desarrollo de redes europeas de intercambio de buenas prácticas y de cooperación, constituyéndose en un componente significativo de la "Europa de las regiones" o "Europa como proceso de aprendizaje" y representando una alternativa a la Unión Europea concebida meramente como entidad burocrática, como mercado, o como moneda única (Boulin y Mückenberger, 1999) ${ }^{7}$.

Pese a su potencial, la limitación más preeminente de las políticas de tiempo en relación a las desigualdades, las relaciones de poder y los procesos de empoderamiento surge de la persistencia de la centralidad de la lógica productivista-mercantil y del "tiempo de reloj" (Adam, 1999) en la organización socio-temporal de las sociedades contemporáneas, así como de su impacto a la hora de articular los distintos tiempos sociales en la vida cotidiana, sin incurrir en mayores cotas de desigualdad. Ésta es, probablemente, una de las razones por las que las políticas de tiempo de trabajo no hayan tenido el desarrollo esperado. Asimismo, si bien se puede afirmar que las políticas de tiempo de la ciudad superan en cierto sentido el determinismo productivistamercantil de las políticas de tiempo de trabajo, a la hora de evaluar su impacto

\footnotetext{
${ }^{7}$ Sirva de ejemplo la consolidación en Barcelona en Febrero de 2009 de la Red Europea de Ciudades con el fin de avanzar hacia una definición conjunta de políticas de tiempo y su implementación (http://www.jornadausosdeltemps.net/es/red ciudades Consultada el 31/05/2012), así como la existencia de una red de universidades que trabajan aspectos relacionados con las políticas de tiempo de forma interdisciplinar (Boccia, 2003:7, Bonfiglioli,
} 2008:94). 
efectivo, se ha apuntado que no consiguen romper con la división sexual del trabajo ni con las desigualdades de género, principalmente en relación al reparto de la carga global de trabajo (Torns, Borrás, Moreno y Recio, 2008).

\section{4) Autonomía, Dependencia Y VULNERABILIDAD}

El segundo eje de los debates actuales sobre los cuidados y la sostenibilidad de la vida al través del que resulta relevante ahondar en torno a la propuesta de ley italiana y a las políticas de tiempo es el relacionado con el cuestionamiento de la dicotomía autonomía/dependencia. Dicha dimensión sale a la luz en torno a la noción bidireccional de los cuidados asumida por la propuesta de ley, así como respecto a la pertinencia del tiempo del ciclo vital y del cuerpo. No obstante, si bien es una dimensión con un gran potencial, resulta uno de los aspectos menos desarrollados en torno a las políticas de tiempo.

Esta mirada pone el foco de atención en la noción de tiempo encarnado: un tiempo que está incorporado a en las personas y que es definitorio de ellas. Es el tiempo del cuerpo, de la edad, del ciclo vital (Adam, 1995; Leccardi, 1996; Ramos, 2007, 2011; Legarreta, 2008, 2013c). Abordar el tiempo incorporado desde una perspectiva sociológica y en relación a los procesos sociales supone una apertura a un campo de estudio no suficientemente explorado hasta el momento y omitido por las primeras propuestas descritas por Emilie Durkheim en torno al tiempo social. Conlleva, asimismo, partir de una premisa que no resulta trivial: "el cuerpo temporalizado es también una construcción social" (Ramos, 2011: 83).

Desde la preeminencia del tiempo encarnado, asumir una concepción bidireccional de los cuidados -en la que todas las personas tienen el derecho y 
la capacidad tanto de prestarlos como de recibirlos- implica al menos dos cuestiones que merece traer a colación.

Por un lado, rompe con la diferenciación entre una parte de la población que precisa cuidados y otra que los presta, por lo que se desvanece la idea de la autonomía plena, al tiempo que se pone de manifiesto el carácter imprescindible del trabajo doméstico y los cuidados para la subsistencia humana: para sobrevivir es preciso que estén cubiertas las necesidades biológicas (sueño, comida, aseo...), así como las afectivas, la seguridad psicológica, y la creación de relaciones y lazos humanos; "estos últimos se consideran tan esenciales para la vida como el alimento más básico" (Carrasco 2001: 14-15). De esta forma, se relaciona con la apuesta por la revalorización del trabajo doméstico y los cuidados señalada en el apartado anterior.

Por otro lado, prima una visión diacrónica de los cuidados, pues se pone de manifiesto que todas las personas los precisan a lo largo de toda la vida y no solamente en momentos determinados (aunque se intensifiquen en situaciones de enfermedad y al inicio y final de la vida). La iniciativa propone, por tanto, enfatizar la vulnerabilidad como característica que atraviesa todas las etapas vitales, a la par que ofrece la posibilidad de aprehender algunas dimensiones relevantes del cuidado que resulta complicado percibir con una mirada sincrónica, puesto que, tal como subrayan otras autoras, las relaciones sociales establecidas en torno a la dependencia y la vulnerabilidad se despliegan a través de un tiempo prolongado, a menudo el tiempo de toda una vida (Dammame, 2011; Dammame y Papperman, 2004). Se invita, por tanto, a una reflexión en torno a la organización social del cuidado, explorada en el siguiente epígrafe.

En esta línea, la iniciativa italiana defiende un modelo de trayectoria vital en el que sea posible compaginar la etapa escolar y el trabajo retribuido, y en el que se puedan tener momentos para la formación y el estudio, excedencias 
parentales y familiares y por asuntos propios en el transcurso de la vida laboral. Con tal finalidad, en la propuesta se proponen medidas que permitan a mujeres y hombres separase temporalmente del mercado para estudiar, para ocuparse de sus hijas e hijos, para cuidar de una persona enferma, para el trabajo comunitario o, simplemente, para tener tiempo para sí. El desarrollo efectivo de tales medidas, sin embargo, ha resultado más bien escaso, dado que hoy por hoy, la desregulación, flexibilización y precarización del mercado laboral plantea obstáculos importantes a la posibilidad de modular el tiempo de trabajo del mercado a la largo del ciclo vital en la línea inicialmente planteada por la propuesta de ley.

No obstante, pese a que su implementación no haya resultado ser la que cupiera esperar, partir de una noción de cuidado de las características que se vislumbran en la iniciativa italiana -diacrónica, universal y necesariamente unida de la existencia humana- encierra un gran potencial tanto desde la perspectiva de la organización social y las relaciones interpersonales, como a nivel individual y encarnado.

En relación a la organización social, una noción de cuidado diacrónica, universal y unida a la existencia humana lleva implícita una definición de los derechos sociales desde una perspectiva individual e inclusiva basada en un nuevo pacto social entre géneros (Lewis, 2007/2011) que cuestiona la centralidad de los derechos ligados a la norma social de empleo (Prieto, 2000, 2007 y García Diez, 2003), así como de aquellos que derivan de la posición de cada individuo en la familia (Pateman, 1988/1995). Personas y colectivos que se encuentran en los márgenes de la estructura social, porque no participan en el mercado laboral o no tienen capacidad de consumo (por ser mayores o demasiado jóvenes, tener diversidad funcional o, simplemente, contar con otras prioridades en su vida) se definen, de esta forma, por su capacidad a la hora de procurar la subsistencia y el bienestar de otras personas. En esta línea se sitúan, por ejemplo, las reivindicaciones más recientes del Foro de la Vida 
Independiente y Divertad que tienen como objetivo la plena consecución de la dignidad y la libertad por parte de las personas con diversidad funcional ${ }^{8}$.

Subrayar la capacidad de todas las personas, independientemente de sus circunstancias y particularidades, conlleva paralelamente aceptar y asumir la propia vulnerabilidad en tanto que característica intrínseca de lo humano. Se puede afirmar, por tanto, que la propuesta italiana asume una noción universal, inclusiva y extensa de la vulnerabilidad, de modo que presenta un vínculo interesante con algunas de las propuestas más relevantes en torno a la ética del cuidado ${ }^{9}$ que "reconocen de manera más realista de lo que lo hacen las teorías sociales y morales «mayoritarias» que la dependencia y la vulnerabilidad no son accidentes del camino, que solo les pasan a los demás (a los «otros»)", sino que son rasgos inherentes a la condición humana (Paperman, 2004: 427). Así la vulnerabilidad se vuelve un atributo que iguala a todas las personas, pasa a formar parte del patrimonio de lo común (L. Gil,

\footnotetext{
${ }^{8}$ El Foro de Vida Independiente y Divertad tiene su origen a mediados del año 2001 con el objetivo de impulsar en el Estado español el movimiento de Vida Independiente surgido en Estados Unidos en 1972. Se define como una comunidad virtual de "reflexión filosófica y de lucha por los derechos de las personas con diversidad funcional" (http://www.forovidaindependiente.org/ Consultado el 30/05/2012). Se propone el término diversidad funcional para hacer referencia al colectivo de personas que es discriminado bien por poseer "cuerpos que tienen órganos, partes del cuerpo o su totalidad que funcionan de otra manera porque son internamente diferentes", bien por ser "mujeres y hombres que por motivos de la diferencia de funcionamiento en su cuerpo realizan tareas habituales (desplazarse, leer, agarrar, vestirse, ir al baño, comunicarse, entre otros) de manera diferente" (Romañach y Lobato, 2007:330).

${ }^{9}$ La ética del cuidado se basa en el desarrollo de disposiciones morales más que en el aprendizaje de principios y privilegia unas respuestas contextuales y específicas así como el punto de vista del "otro concreto". Esta perspectiva es desarrollada, entre otras, por Joan Tronto, que sostiene que la ética del cuidado no es una disposición moral específica de las mujeres, sino un efecto de su posición social subalterna (Molinier, 2011) y reivindica su potencial como herramienta política (Papperman, 2011; Molinier, 2011). La ética del cuidado ha sido criticada por su esencialismo, advirtiendo que tanto desde una perspectiva histórica (a lo largo del tiempo), antropológica (en relación a otras culturas) como sociológica (desde una mirada de clase) se puede apreciar que los atributos que se asocian con el cuidado se definen en relación a una forma específica de entender la maternidad (Torns, 2007) que toma como referencia a la mujer desde una perspectiva biológica, considerándola, en tanto que sujeto político, como grupo homogéneo (Artiaga, 2009). Dichas críticas son asumidas por autoras que se posicionan a favor de la perspectiva de la ética del cuidado pero que se desvinculan de dicha forma específica de entenderla, pues la asocian principalmente a una corriente "maternalista", ejemplificada en el trabajo de Nel Noddings, que define la ética del cuidado como "ética femenina" y no como "ética feminista" (Papperman, 2011: 39).
} 
2011; Papperman, 2011): cada uno/a podemos reconocernos en el otro/a a través de nuestra propia condición inestable, precaria en parte, contingente, circunscrita al entorno que nos rodea, e interdependiente. Frente al utilitarismo e individualismo de la lógica mercantil, cabe abogar por unas relaciones interpersonales basadas en el principio de reciprocidad (Legarreta, 2013b)

Desde un posicionamiento tal, la autonomía no se define como autosuficiencia sino como "capacidad de los individuos de sustraerse del poder, ser de un modo más propio, decidir sobre la vida" (L. Gil, 2012). Una vez más, se cuestiona el modelo de organización social hombre ganador de pan-mujer ama de casa que, a través del salario familiar, otorga un estatus privilegiado al varón en la familia y en la sociedad como sustentador de los miembros del hogar. Asimismo, sale a la luz la necesidad de cuidados que ha sido invisibilizada por el mito de autonomía plena del hombre trabajador, caricaturizado en la figura del "trabajador champiñón" (Peréz Orozco y del Río, 2002 y Pérez Orozco, 2010: 137): aquel que brota todos los días plenamente disponible para el mercado, sin necesidades de cuidado propias ni responsabilidades de cuidado ajenas, y desaparece una vez fuera de la empresa. Se quiebra la imagen del homo economicus y en su lugar toma sentido y fuerza la del "homo vulnerabilis" (Molinier, 2011: 59) como único modelo de lo humano.

Paralelamente, al desdibujar la dicotomía autonomía/dependencia se pone de manifiesto la precariedad de la propia existencia pues, como apunta Pascale Molinier, "no resulta posible reconocer la vulnerabilidad del otro sin movilizar la propia sensibilidad" (Molinier, 2011: 58$)^{10}$. De este modo, se vuelve

\footnotetext{
${ }^{10}$ Pascale Molinier desarrolla una reflexión en torno al trabajo de las enfermeras, basándose en la narración de sus experiencias (Molinier, 2008, 2011) que resulta especialmente interesante pues abre una vía a la des-naturalización de los cuidados en un doble sentido: da relevancia a la presencia de sentimientos de rechazo (que se podrían identificar como "contrarias" a lo que se supone que es el cuidado) y muestra el cuidado como un aprendizaje, como "un saber adquirido por la experiencia" (Molinier, 2011:55). Así, logra desligar el cuidado de la feminidad, al tiempo que disipa toda tentativa de idealización. 
pertinente abordar una de las dimensiones del cuidado a menudo olvidada: el autocuidado. Frente a la fragilidad de la existencia, tan importante como ser cuidadas/os es cuidarse a sí misma/o. Se apela, de esta forma, a la dimensión individual del cuidado y la sostenibilidad de la vida, una mirada micro inevitablemente interpelada por los límites de la estructura y del imaginario social. La posibilidad de procurar el bienestar propio no coincide con los ideales de feminidad constituidos en la Modernidad a través del discurso de la domesticidad y heredados en la actualidad (Legarreta, 2007). Para cuidarse a sí misma es preciso apropiarse de un tiempo para sí, un tiempo propio, y la domesticidad difícilmente contempla dicha posibilidad (Murillo, 1996). La otra cara de la moneda la constituye la figura masculina del "trabajador champiñón". En este caso, el autocuidado hace referencia al desempeño por parte de uno mismo de las tareas domésticas básicas e imprescindibles para procurar la propia subsistencia y cubrir los umbrales mínimos de bienestar (comida, limpieza, aseo...).

\section{5) MÁS ALLÁ dE LA CONCILIACIÓN: HACIA UNA NUEVA ORGANIZACIÓN SOCIAL}

El tercer eje de los debates actuales sobre los cuidados y la sostenibilidad de la vida que resulta oportuno abordar en torno a la propuesta de ley italiana y a las políticas de tiempo es el referente a la estructuración y el cambio social. En este caso, el tiempo sirve como instrumento para la transformación social de cara a elaborar propuestas para avanzar hacia una organización social más justa y equitativa. Desde esta perspectiva, sale a la luz su dimensión performativa. El tiempo no sólo posee la capacidad de definir la realidad sino también de transformarla (Ramos, 2005): nuevas formas de definir el tiempo y la organización temporal pueden ofrecer nuevas formas de entender la sociedad y vivir en ella. 
En referencia a la estructuración y el cambio social, los debates en torno a los cuidados y la sostenibilidad de la vida centran la atención principalmente en los límites que plantea la conciliación para apostar por una nueva organización social del cuidado. En este sentido, la propuesta de ley emerge con la pretensión de abordar el tiempo desde una perspectiva multidimensional y situada, para avanzar hacia una nueva organización temporal más equitativa e igualitaria entre géneros y generaciones tanto en el entorno laboral y de participación social, como en el ámbito privado y en el doméstico-familiar. Por ello, se puede afirmar que en sus orígenes las políticas de tiempo van más allá de las políticas de conciliación desarrolladas principalmente con el objetivo de fomentar el empleo femenino (en un contexto de flexibilización y desregularización laboral) e incentivar la natalidad entre la población ocupada, para paliar la baja fecundidad que afecta a los países de la Unión Europea (Torns, 2005 y Torns, Borrás, Moreno y Recio, 2008) ${ }^{11}$. Las políticas de conciliación están dirigidas principalmente a las mujeres con empleo y no ponen en tela de juicio la división sexual del trabajo, ni los roles tradicionales de género. Además, parten de una perspectiva que prioriza el mercado y dirigen la intervención a la regulación de la jornada laboral en base a una definición del trabajo y del tiempo marcadamente mercantilista: el trabajo se reduce a empleo y el tiempo a "tiempo de reloj" (Adam, 1999).

Fundamentalmente, la conciliación no promueve un reparto de tiempos y de trabajos equitativo a nivel social ni fomenta la corresponsabilidad en el ámbito doméstico-familiar. La iniciativa italiana, sin embargo, nace precisamente con dicha pretensión, por lo que parte de una definición amplia del trabajo y una perspectiva plural y multidimensional de tiempo que no se

\footnotetext{
${ }^{11}$ Las políticas de conciliación surgen a partir del año 2000 en el marco general de las Estrategias Europeas de ocupación, dentro del denominado cuarto pilar, cuyo objetivo es la promoción del empleo femenino. En el contexto español, se ha señalado que la denominada Ley de Conciliación de la Vida Familiar y Laboral de las Personas Trabajadoras (Ley 39/1999) no hace más que recoger, integrar y ampliar las normas previamente existentes, pero hasta el momento dispersas (Campillo, 2010:202). 
ciñe a la gestión de horarios: "[h]ablar de tiempo en vez de horario significa reconocer que, además del trabajo y las formas de su organización, hay otros ámbitos de la vida humana a los que dar valor y fuerza" (Cordoni, 1993: 221)

De esta forma, como se ha mencionado anteriormente, la propuesta italiana aboga por la revalorización del trabajo doméstico y de los cuidados para poner el foco de atención en las necesidades de las personas y superar "la concepción de la familia como lugar elegido para satisfacer, a través del trabajo invisible y gratuito de las mujeres, las necesidades y los derechos no reconocidos" (Cordoni, 1993: 225). En esta línea, plantea la necesidad de diseñar actuaciones y medidas que fomenten la corresponsabilidad entre géneros y generaciones en el ámbito doméstico-familiar y, paralelamente, propone el aumento y la universalización de los servicios y recursos dirigidos a cubrir la demanda de cuidados, así como una organización de la jornada laboral que permita a la población con empleo disponer de más tiempo para el ámbito doméstico-familiar, pues se parte de la afirmación de que "cuidarse de los otros, ocuparse de ellos, no es un tipo de trabajo enteramente socializable mediante servicios asistenciales sino una función cada vez más importante y que requiere tiempo" (Sección femenina nacional del PCI, 1989/1990).

Desde este posicionamiento, es posible observar cierta similitud entre la propuesta italiana y las reflexiones más recientes en torno al social care, una propuesta desarrollada "por las analistas británicas de las políticas de bienestar, con el fin de reclamar que el Estado de Bienestar contemple la organización social del cuidado además de las políticas ya existentes" (Carrasco, Borderías, Torns, 2011: 36) ${ }^{12}$. El social care hace referencia a las actividades implicadas en satisfacer las demandas físicas y emocionales de adultos/as y niños/as, así como la normatividad, los costes y los contextos

\footnotetext{
12 El social care es una noción desarrollada por Jane Lewis (1998) y Mary Daly y Jane Lewis (2000/2011). Pilar Carrasquer (2013) subraya la virtud de la propuesta a la hora de proponer un concepto teórico con suficiente capacidad explicativa como para alcanzar un campo de estudio.

Matxalen Legarreta 
sociales dentro de los cuales este trabajo es asignado y llevado a cabo. Propone una aproximación que contempla una doble vertiente. Por un lado, a nivel macro, pone el acento en la división del trabajo, de la responsabilidad y de los costes del cuidado entre la familia, el mercado, el Estado y la comunidad (sociedad civil). Por otro, a nivel micro, arroja luz sobre el contenido y el contexto del trabajo realizado en los hogares principalmente por las mujeres, así como sobre la distribución del trabajo doméstico y de cuidados entre hombres y mujeres y las condiciones en las que se lleva a cabo. De esta forma, engloba tanto los aspectos materiales-estructurales, como los intersubjetivos, relacionales y simbólicos, relacionados con la organización social del cuidado, ya que, tal como apunta Carrasquer, conceptualiza los cuidados en tanto que "trabajo socialmente organizado en el que participan distintos grupos, instituciones y espacios sociales, que se articula y distribuye de acuerdo a distintos patrones socio-culturales y económicos" (Carrasquer, 2013: 98).

En relación a la organización social del cuidado y al reparto de la carga global de trabajo, la iniciativa italiana se une, además, a las voces que evidencian que, hoy por hoy, la conciliación resulta "imposible" y solo caben los "permanentes malos arreglos" (Torns, Teresa; 2005). Así, comparte la posición defendida, entre otras, por la economía feminista: no resulta viable conciliar dos lógicas con objetivos contrapuestos, la lógica del mercado y la lógica de la sostenibilidad de la vida. Como apunta Cristina Carrasco aludiendo a la oposición entre capital y vida, no es factible llegar a un consenso o a una complementariedad entre ambas, se deben establecer prioridades y dar prioridad a la sostenibilidad de la vida supone, necesariamente, apostar por una nueva organización social (Carrasco, 2003). Dos décadas antes de que Carrasco escribiese su texto, la propuesta italiana suscribe dicha posición:

"la conclusión se impone: hay que volver a barajar y establecer una nueva jerarquía de valores que comprenda criterios sobre qué es importante, qué es público (...), qué es lo que requiere tiempo. (...) Lo que se propone [en la 
propuesta de ley] no es imposible. Obliga, eso sí, a reconocer que el tiempo dedicado a las ocupaciones domésticas es «tiempo social»" (Sección femenina nacional del $\mathrm{PCl}$, 1989/1990)

Desde esta mirada, se puede también vislumbrar una relación interesante entre la propuesta italiana y la reivindicación del derecho a la "cuidadanía", que resume la apuesta política por poner en el centro el cuidado de la vida como responsabilidad social y política, y no como proceso que ha de ser resuelto en los hogares, principalmente bajo la responsabilidad de las mujeres (Pérez Orozco y del Río, 2004 y Pérez Orozco, 2006b).

\section{6) UN EJEMPLO: LOS BANCOS DE TIEMPO}

Los bancos de tiempo son probablemente las iniciativas que mayor eco han tenido en el marco de las políticas de tiempo, tanto en nuestro contexto más cercano, como a nivel internacional ${ }^{13}$. Se implementan como experiencias locales desarrolladas por un conjunto de personas que participan en los mismos con el fin de intercambiar prestaciones y servicios tomando como unidad de valor y de cambio el tiempo. Así, resultan ser un ejemplo práctico de algunos de los aspectos desarrollados en los epígrafes anteriores en relación a los tres elementos clave identificados en los debates sobre los cuidados y la sostenibilidad de la vida: desigualdades sociales y procesos empoderamiento; autonomía, dependencia, vulnerabilidad; y la estructuración y cambio social.

En relación a la erradicación de las desigualdades y la activación de procesos de capacitación y de empoderamiento, cabe señalar que este tipo de iniciativas permite desdibujar las desigualdades tanto entre diferentes grupos sociales, como entre actividades y tiempos. Por un lado, el banco de tiempo es

\footnotetext{
${ }^{13}$ Los primeros bancos de tiempo comienzan a funcionar en Estados Unidos en la década de 1980 tomando como referente la idea de time dollar de Edgar Cahn. En Europa ha sido pionera Italia, donde se han consolidado de tal forma que cuentan con un observatorio nacional (http://www.tempomat.it Consultado el 27/02/2014 ). Se han extendido también por el Reino Unido y Portugal (Gisbert, 2010) y, en el contexto español, si bien los primeros se crean en Barcelona (Recio, Méndez y Altés, 2009), en los últimos años, hay cada vez más experiencias a lo largo de todo el Estado (http://www.vivirsinempleo.org/ Consultado el 03/11/2013). 
un recurso que está al alcance de toda la población ${ }^{14} \mathrm{y}$, a diferencia de los bancos del ámbito financiero, en el que todos los «clientes» son iguales: son portadores de recursos y tienen, a su vez, necesidades que cubrir (Torns, 2001). Población mayor o adolescente, personas con diversidad funcional ${ }^{15}$, población desempleada 0 migrante $^{16}$ puede redefinirse a sí misma como productora y contribuyente, y no simplemente como receptora de ayuda. Además, generan una actividad situada y contextualizada que sirve para reforzar los vínculos comunitarios por lo que, a menudo, se desarrollan con la colaboración de instituciones locales y redes sociales, a través de estrategias de investigación-acción-participativa ${ }^{17}$. Por otro lado, al emplear una magnitud estandarizada de tiempo como unidad de intercambio (las horas) se dota a todas las prestaciones y servicios del mismo valor. Cada actividad y ocupación se intercambia por el tiempo que se tarda en llevarla a cabo. Mediante los bancos de tiempo, el trabajo doméstico y los cuidados cobran el mismo valor que las actividades con mayor prestigio social (formación, asesoría y gestión, por ejemplo), por lo que se logra hacer visible el trabajo desempeñado tradicionalmente por las mujeres en los entornos doméstico-familiares, dotarlo de valor y promover su reconocimiento social.

\footnotetext{
${ }^{14}$ Se caracterizan por una forma de participar que no presenta grandes dificultades: cada participarte dispone de un listado informativo con las ofertas y demandas de los y las demás participantes para poder seleccionar los servicios, identificar a quienes los ofrecen, ponerse en contacto con ellos/as, y concretar el intercambio (Méndez y Altés, 2009).

${ }^{15}$ El banco de tiempo de la Universidad de Sevilla cuenta con un voluntariado de "abuelos/as adoptivos/as". Asimismo, para facilitar el acceso a las personas con diversidad funcional, su página web ofrece un video realizado en lenguaje de signos (http://www.sacu.us.es/es/06 03.asp Consultada el 21/10/2013).

${ }^{16}$ Puede servir de ejemplo la experiencia del banco del tiempo de la Fundación Altauri en los barrios de Bilbao La Vieja, San Francisco y Zabala de Bilbao, emblemáticos por la elevada presencia de población migrante. Esta iniciativa ha sido pionero en traducir y adecuar un programa informático para la gestión de bancos de tiempo que cede de forma gratuita a asociaciones sin ánimo de lucro (http://www.bdtbilbao.org/index.php Consultada el 21/10/2013).

${ }^{17}$ Es el caso del banco de tiempo del barrio Pozokoetxe de Basauri (Bizkaia) inaugurado el 15 de Marzo de 2012. Si bien la iniciativa es impulsada en un primer momento por el Área de Igualdad del Ayuntamiento cuenta con un extenso trabajo de reflexión colectiva en el barrio (http://www.equitruke.net/bdt eusk/index.php Consultada el 21/10/2013).
} 
Respecto al cuestionamiento de la dicotomía autonomía/dependencia, en tanto que sistemas de intercambio, los bancos de tiempo incentivan el reconocimiento de la propia vulnerabilidad que sale a luz a la hora de demandar una prestación o servicio, pues no siempre resulta fácil pedir ayuda. Paralelamente, promueven una mejora de la calidad de vida, de la autoestima y de la autorrealización, ya que sirven no sólo para intercambiar actividades necesarias para la vida cotidiana, sino también aquellas que proporcionan bienestar y satisfacción (Méndez y Altés, 2009) (la manicura o un masaje, por ejemplo).

En relación a la estructuración y el cambio social, el banco de tiempo se caracteriza por su carácter instrumental en tanto que herramienta que no se crea como objetivo en sí mismo, sino como vehículo para lograr otros objetivos: crear y reforzar lazos sociales promoviendo la reciprocidad y la paridad; fomentar la capacitación y el empoderamiento de la población a través del reconocimiento de la propia vulnerabilidad; y dotar del mismo valor y reconocimiento a todas las actividades y ocupaciones de la vida cotidiana, poniendo de manifiesto la relevancia social del trabajo doméstico y de cuidados. Por ello, los intercambios tienen carácter multilateral y multirecíproco $^{18}$, y se definen por su eventualidad, no se pretende reemplazar la labor de los servicios sociales, pues no se requiere profesionalización por parte de las personas que prestan los servicios (Méndez y Altés, 2009). Asimismo, los bancos de tiempo representan una alternativa a la linealidad de la acumulación de beneficios, pues funcionan en base al principio circular de reciprocidad (dar, recibir y devolver) más próxima a la lógica de la satisfacción de las necesidades humanas (Legarreta, 2013b).

\footnotetext{
${ }^{18}$ Las personas participantes en el banco de tiempo ofrecen uno o varios servicios a la totalidad de participantes y solicitan el servicio que necesitan a la persona que lo ofrece, sin ser necesariamente la misma de la que ha recibido un servicio anteriormente. 
Si bien se puede constatar que los bancos de tiempo son herramientas interesantes, presentan también ciertos inconvenientes: son "las luces y sombras" de este tipo de proyectos (Del Moral, 2013). Concretamente, cabe señalar al menos tres problemas en relación a los bancos de tiempo: reproducción de los roles de género, reproducción de privilegios, y reproducción del tiempo como "tiempo de reloj".

Por un lado, en cuanto a la reproducción de los roles de género, se ha de advertir que los bancos de tiempo se convierten frecuentemente en redes de intercambio entre mujeres, de modo que no se cuestionan los roles tradicionales, ni se socializa el reparto de la cobertura de las necesidades cotidianas. Por tanto, es posible que lleguen a generar redes de solidaridad y de apoyo entre mujeres, así como a reforzar o formalizar las ya existentes, lo que puede implicar la minimización u ocultación del malestar de muchas de ellas, que tienen dificultades a la hora de hacer frente a su sobrecarga de trabajo en solitario.

Por otro lado, respecto a la reproducción de privilegios, los bancos de tiempo descansan sobre la noción de tiempo como recurso: el tiempo es tomado como algo de lo que se dispone para actuar, lo que implica que la acción precisa tiempo, que este tiempo que se precisa se ha de tener y, que para tenerlo, es necesario que previamente esté disponible (Ramos, 2007). Por tanto, para poder intercambiar tiempo, es necesario tenerlo disponible, de modo que las personas que no disponen de él (por estar desempeñando labores de cuidado de forma intensiva, por ejemplo) no pueden llegar a intercambiarlo, si bien precisamente por ello quizá sean las que mayor necesidad tengan de participar en un red de este tipo. El tiempo puede ser intercambiado únicamente por las personas "privilegiadas" que disponen de un cómputo extra de tiempo de libre disposición más allá de sus obligaciones diarias (sean éstas remuneradas o no). 
Por último, en relación a la reproducción del tiempo como "tiempo de reloj", los bancos de tiempo descansan sobre una paradoja: se crean como un modo alternativo de intercambio que funciona al margen del mercado, pero la concepción de tiempo sobre la que descansan está ineludiblemente ligada a la lógica productivista-mercantil. El intercambio se realiza tomando como unidad de valor y de cambio la duración de las actividades en tanto que magnitud cuantificable. Es una unidad abstracta y estandariza, muy arraigada en las sociedades occidentales contemporáneas y, por tanto, familiar y fácilmente reconocible por cualquier persona: horas y minutos. De este modo, se facilita y se promueve la participación de todas las personas en los bancos de tiempo, pues el intercambio se realiza a través de algo que es por todas y todos conocido, y habitualmente empleado. Cabe advertir, sin embargo, que dicha forma de entender el tiempo no es universal ni a-histórica, sino que surge y se consolida durante el siglo XIX a través de los procesos de industrialización y urbanización como parte constituyente del avance y afianzamiento del capitalismo industrial (Thomson, 1979; Le Goff, 1987). Supone una forma de pensar y utilizar el tiempo que responde a una concepción mercantilista del trabajo, de la economía y, por extensión, de las relaciones humanas (Adam, 1999).

A pesar de su ineludible sesgo mercantilista, la utilización del tiempo cronométrico como unidad de valor y de cambio resulta la forma más factible de poder establecer relaciones de intercambio equitativas, pues al emplear una magnitud abstracta, homogénea y estandarizada para estimar el valor de cada actividad, se neutralizan los matices con los que nace y se desarrolla, así como sus particularidades, y se disuelven las jerarquías entre tiempos y ocupaciones. Recurrir a una concepción de tiempo como "tiempo de reloj" plantea, por tanto, un dilema de difícil solución. Por un lado, surge el peligro de naturalizar este modo de concebir el tiempo, limitando así la posibilidad de pensar otras temporalidades, otras lógicas temporales, y otras formas de temporalizar las 
relaciones sociales, más allá de la productivista-mercantil (Legarreta, 2008). Por otro lado, sólo recurriendo a la cuantificación se hace viable equiparar los tiempos de trabajo doméstico y de cuidado con otros tiempos de mayor prestigio social para poder avanzar hacia su revalorización y reconocimiento ${ }^{19}$.

\section{7) CONCLUSIONES}

A lo largo del texto se ha intentado sacar a la luz el potencial de las políticas de tiempo a la hora de abordar las relaciones sociales desde la centralidad de los cuidados y la sostenibilidad de la vida, concretamente a través de la propuesta de ley de iniciativa popular desarrollada en Italia a finales de 1980. El tiempo ha servido de vehículo para llevar a cabo dicha reflexión en torno a algunos de los aspectos medulares que configuran los debates actuales sobre los cuidados y la sostenibilidad de la vida: desigualdades sociales y procesos de empoderamiento; autonomía, dependencia, vulnerabilidad; y estructuración y cambio social. Asimismo, tal reflexión se ha visto reflejada en un ejemplo concreto: los bancos de tiempo.

Con todo ello, de forma trasversal, se han desarrollado en el texto tres aspectos que resultan claves a la hora de afianzar una reflexión sobre la vida social que ponga el foco de atención en las dinámicas de lo cotidiano y en los procesos de satisfacción de las necesidades humanas: equilibrio entre las dimensiones materiales y no-materiales, interrelación entre la mirada micro y macro, e intento de superación del pensamiento dicotómico.

En primer lugar, la propuesta italiana aboga por un equilibrio entre las dimensiones materiales y no-materiales de la vida social. Por un lado, da cuenta de las estructuras sociales que se materializan temporalmente en calendarios, horarios y jornadas; pone el foco de atención en las ocupaciones que es preciso llevar a cabo para garantizar la satisfacción de las necesidades

\footnotetext{
${ }^{19}$ Esta misma disyuntiva emerge al analizar el empleo de las Encuestas de Usos de Tiempo para cuantificar el volumen de trabajo doméstico y cuidados y su desigual distribución (Legarreta, 2005, 2008, 2010) 
humanas (definidas como trabajo) y en el tiempo que es preciso emplear para cubrirlas; y señala la pertinencia de tener en cuenta la materialidad del cuerpo, del ciclo vital y de las edades. Por otro, desde la distinción entre tiempo y horario, deja clara la relevancia de la dimensión intersubjetiva, de las significaciones situadas y contextualizadas: "hablar de tiempo en vez de horario (...) no es un sutil distinción filosófica. Los dos términos encierran una diferencia profunda y concreta: el horario es una magnitud cuantitativa, medida y establecida de la jornada, que guía una parte de la vida, el tiempo conlleva, por el contrario, una dimensión subjetiva personal” (Cordoni, 1993: 221).

En segundo lugar, la propuesta italiana desarrolla una reflexión que, si bien tiene como objetivo dar valor a lo cotidiano, a las esferas más esenciales de la vida, no descuida los procesos y las relaciones propias del ámbito macroestructural. Se apuesta, en este sentido, por poner el énfasis en los procesos y las dinámicas del día a día, en el cuerpo y en el ciclo vital, una mirada micro atentamente interrelacionada con el tiempo macro-estructural del mercado laboral y de la ciudad. Los tres apartados en los que se divide la propuesta de ley (ciclo vital, tiempo de trabajo y tiempo de la ciudad) permiten contemplar la vida social con una lente u otra, según el foco con el que se quiera observar, para ofrecer un panorama global.

En tercer lugar, al partir de una noción de tiempo plural, no-jerárquica, encarnada y unida a la experiencia, la iniciativa supera el pensamiento dicotómico para ofrecer una reflexión que abarca el tiempo desde diferentes ángulos: como se ha señalado anteriormente, cada apartado en el que se divide la propuesta de ley hace referencia a una dimensión distinta del tiempo, pero complementaria con el resto. Así, se acerca a la imagen de una "tela de araña" (Leccardi, 1996) en la que cada elemento tiene sentido en sí mismo, a la par que contribuye a la composición del diseño global. En este sentido, cuando se da cuenta de una dimensión del tiempo u otra se trata de más bien de "enfocar y no de aislar" (Adam, 1990: 462). 
CEIC

\section{8) Bibliografía}

Adam, Barbara (1989) "Feminist social theory needs time. Reflections on the relation between feminist thought, social theory and time as an important parameter in social analysis". Sociological Review, 37 (3): 458-473.

Adam, Barbara (1995) Timewach. The social analysis of time. Cambridge. Polity Press.

Adam, Barbara (1999) "Cuando el tiempo es dinero. Racionalidades de tiempo conflictivas y desafíos a la teoría y la práctica del trabajo". Sociología del trabajo, Nueva Época, 37: 5-39.

Artiaga, Alba (2009) La producción política de la categoría de cuidados: voces de los movimientos feministas y marcos interpretativos de la Ley de Dependencia. I Congreso Anual de la Red-ESPANET: Treinta años de Estado de Bienestar en España. Logros y retos para el futuro. Oviedo 57 de noviembre. Disponible en: http://www.espanetspain.net/congreso2009/archivos/ponencias/TP11P01.pdf Consultado el 21/06/2012.

Balbo, Laura (1990) "Una ley para las horas futuras". Mientras tanto, 42: 59-63

Balbo, Laura (ed.) (1991) Tempi di vita. Milán: Giangiacomo Feltrinelli.

Beck, Ulrich y Beck-Gernsheim, Elisabeth (2001a) El normal caos del amor. Las nuevas formas de la relación amorosa. Barcelona: Paidós.

Beck, Ulrich y Beck-Gernsheim, Elisabeth (2001b) "De «vivir para los demás» a "vivir la propia vida»: la individualización y la mujer", en La individualización. El individualismo institucionalizado y sus consecuencias sociales y políticas. Barcelona: Paidós [2003].

Boccia, Teresa (2003) El tiempo y el espacio de las ciudades: la experiencia italiana. Segundo Seminario Internacional Sobre Género y Urbanismo. Infraestructuras para la Vida Cotiana ETSAM, UPM. 27 y 28 de mayo de 2002. Publicación ALM en http://www.generourban.org

Bonfiglioli, Sandra (2008) "El Plan territorial del tiempo. Políticas y proyectos sobre los tiempos de la ciudad". Revista Papers, 49: 93-101.

Bonfiglioli, Sandra (2009) Las transformaciones urbanas: impacto sobre el tiempo y el horario. Jornada Europea ¿Hacia dónde va nuestro tiempo? 4 y 5 de Febrero. Ayuntamiento de Barcelona. Barcelona. Disponible en: http://jornadausosdeltemps.net/docs/pon bonfiglioli.pdf. Consultado el 22/04/2012.

Boulin, Jean Yves (2006) "Local time policies in Europe" en Diane Perrons, Colette Fagan, Linda McDowell, Kath Ray y Kevin Ward, (ed.) Gender divisions and the working time in the New Economy. Changing patterns 
of work, care and public policy in Europe and Norh America. Edward Elgar Publishing, 193-206.

Boulin, Jean Yves y Mückenberger, Ulrich (1999) Tiempo de la ciudad y calidad de vida. Fundación Europea para la Mejora de las Condiciones de Vida y de Trabajo.

Campillo, Inés (2010) "Políticas de conciliación de la vida laboral y familiar en los regímenes de bienestar mediterráneos: los casos de Italia y España". Política y Sociedad, 47 (1): 189-213

Carrasco, Cristina (ed.) (2001) Tiempo, trabajos y género. Barcelona: Publicaciones de la Universitat de Barcelona.

Carrasco, Cristina (2003) “¿Conciliación? No, gracias. Hacia una nueva organización social" en Grup Dones i Treballs, Malabaristas de la vida. Mujeres, tiempos y trabajos. Barcelona: Icaria: 27-51.

Carrasco, Cristina; Borderías, Cristina; Torns, Teresa (2011) "Introducción" en El trabajo de cuidados. Historia, teoría y política. Madrid: Catarata.

Carrasquer, Pilar (2013) "El redescubrimiento del trabajo de cuidados, algunas reflexiones desde la sociología". Cuadernos de Relaciones Laborales 31(1): 91-113.

Cordoni, Elena (1993) "Las mujeres cambian los tiempos". Cuaderno de Relaciones Laborales 2: 221-237.

Daly, Mary y Lewis, Jane (2000) “El concepto de 'social care' y el enálisis de los estados de bienestar contemporáneos" en Cristina Carrasco, Cristina Borderías y Teresa Torns (eds.) (2011) El trabajo de cuidados. Historia, teoría y políticas. Madrid: Catarata, 225-251.

Damamme, Aurelie (2011) "El care en las familias: perspectiva temporal verus radiografía" en Luz Gabriela Arango y Pascale Molinier (comp.) El trabajo y la ética del cuidado. Medellín: La Carreta.

Damamme, Aurelie y Papperman, Patricia (2009) "Care domestique: de histories sans début, sans milieu et sans fin". Multitudes, 37: 98-105.

Davies, Karen (1994) "The tensions between process time and clock time in care-work. The example of day nurseries". Time and Society, 3 (3): 277303.

Del Moral, Lucía (2012) "Sobre la necesaria reorganización social de los tiempospolíticas de tiempo, espacios económicos alternativos y bienestar". Papeles de relaciones ecosociales y cambio global, 119: 7791.

Del Moral, Lucía (2013) "Luces y sombras de los espacios comunitarios de intercambio para la generación de bien-estar: los bancos de tiempo ¿una herramienta feminista?" Comunicación presentada en el IV Congreso de Economía Feminista, Sevilla, 3-5 de Octubre. Disponible 
en:

http://riemann.upo.es/personal-wp/congreso-economiafeminista/files/2013/10/delMoral Lucia.pdf Consultado el 11/12/2013.

Esquivel, Valeria (2009) La "economía del cuidado": un recorrido conceptual. Universidad Nacional de General Sarmiento. Consejo Nacional de Mujeres República Argentina. Disponible en: http://www.cnm.gov.ar/generarigualdad/attachments/article/225/La econ omia del cuidado.pdf. Consultado el 05/06/2012.

García Díez, Susana (2001) "La integración contable del trabajo doméstico. La experiencia española de la última década del siglo XX" en Cristina Carrasco (ed.) Tiempo, trabajos y género. Barcelona: Publicaciones de la Universitat de Barcelona.

García Díez, Susana (2003) "La ciudadanía laboral: una reinterpretación socioeconómica de las fronteras del sistema de nacional de cuentas". Cuadernos de Relaciones Laborales, 21 (1): 167-178.

Garro, Ohiana (en prensa) "El arte y la construcción del sujeto: una reflexión con Nan Goldin acerca de las narrativas familiares". Arte, Individuo y Sociedad, 26 [mimeo cedido por la autora]

Gisbert, Julio (2010) Vivir sin empleo. Trueque, bancos de tiempo, monedas sociales y otras alternativas. Barcelona: Los libros del lince.

Grau, Elena (1990) "El tiempo es un perro que muerde sobre todo a las mujeres". Mientras tanto, 42: 43-44.

Junco, Carolina; Pérez Orozco, Amaia y Del Río, Sira (2004) "Hacia un derecho universal de cuidadanía (sí, de cuidadanía)". Libre pensamiento, 51: 4449.

Leccardi, carmen (1996) "Rethinking social time: feminist perspectives". Time and Society, 5 (2): 169-186.

Legarreta, Matxalen (2005) "Cuantificación de la cotidianidad: Las Encuestas de Usos del Tiempo como instrumento de medida". Revista Vasca de Sociología-Inguruak, 41:87-98

Legarreta, Matxalen (2007) "La globalización del trabajo doméstico y de cuidados. Nuevas configuraciones de la domesticidad", en Jesús Arpal e Ignacio Mendiola (eds.): Estudios sobre cuerpo, tecnología y cultura. Servicio Editorial de la Universidad del País Vasco.

Legarreta, Matxalen (2008) "El tiempo donado en el ámbito doméstico. Reflexiones para el análisis del trabajo doméstico y los cuidados". Cuadernos de Relaciones Laborales, 26(2): 45-69.

Legarreta, Matxalen (2010) Tiempo y desigualdades de género: Distribución social y políticas de tiempo. Sevilla: Instituto Andaluz de la Mujer.

Legarreta, Matxalen (2013a) "El tiempo donado en el ámbito domésticofamiliar: distribución social, moralización del tiempo y reciprocidad". 
Comunicación presentada en el XI Congreso Español de Sociología, celebrado en Madrid el 10, 11 y 12 de Julio.

Legarreta, Matxalen (2013b) "El tiempo como herramienta para la economía feminista. (Una propuesta a partir del estudio del trabajo doméstico y los cuidados)". Comunicación presentada en el IV Congreso de Economía Feminista, Sevilla, 3-5 de Octubre. Disponible en Internet: http://riemann.upo.es/personal-wp/congreso-economia-

feminista/files/2013/10/Legarreta-Matxalen.pdf Consultado

el $11 / 12 / 2013$.

Legarreta, Matxalen (2013c) "Care crisis and its temporal features: embodied time, moral time and political time". Comunicación presentada en el II Seminario Internacional "Tempos Sociais e o Mundo Contemporâneo" celebrado en Braga (Portugal) el 19 y 20 de Noviembre.

Le Goff, Jacques (1987) "Tiempo de la iglesia y tiempo del mercader en la edad media" y "El tiempo del trabajo en la "crisis" del siglo XIV: Del tiempo medieval al tiempo moderno", en Tiempo, trabajo y cultura en el Occidente medieval. Madrid: Taurus.

L. Gil, Silvia (2011) Nuevos feminismos. Sentidos comunes en la dispersión. Una historia de trayectorias y rupturas en el Estado español. Madrid: Traficantes de sueños.

L. Gil, Silvia (2012) "No existe vida posible sin el vínculo con los otros" Entrevista publicada en Diagonal Web, 9 de Abril, núm. 171, 172. Disponible en: http://www.diagonalperiodico.net/No-existe-vida-posiblesin-el.html. Consultado el 22/04/2012.

Lewis, Jane (1998) "Gender, Social Carea n Welfare Regimes". Journal of European Social Policy, 2 (3): 159-173.

Lewis, Jane (2007) "Género, envejecimiento y el 'nuevo pacto social': la importancia de desarrollar un enfoque holístico de las políticas de cuidados" en Cristina Carrasco; Cristina Borderías; Teresa Torns (eds.) (2011) El trabajo de cuidados. Historia, teoría y política. Madrid: Catarata, 336-358.

Lombardo, Emauela y Sangiuliano, María (2008) "Género y empleo en los debates político italianos 1995-2007: la construcción de sujetos de género 'no empleados"'. Revista Administración \& Ciudadanía, 3 (2): 105-123. Disponible en: http://www.ucm.es/info/target/Art Chs ES/ArtGeneroEmpleolt AdmCid 08ES.pdf. Consultado el 22/04/2012.

Méndez, Elvira y Altés, Josefina (2009) "Los bancos de tiempo" en Carolina Recio, Elvira Méndez y Josefina Altés, Los bancos de tiempo. Experiencias de intercambio no monetario. Barcelona: Graó, 61-78.

Molinier, Pascale (2008) Trabajo y compasión en el mundo hospitalario. Una aproximación a través de la psicodinámica del trabajo. Cuaderno de 
Relaciones Laborales, Monografía Domesticación del trabajo, 26 (2): 121-138.

Molinier, Pascale (2011) "Antes de todo, el cuidado es un trabajo" en Luz Gabriela Arango y Pascale Molinier (comp.) El trabajo y la ética del cuidado. Medellín: La Carreta.

Mückenberger, Ulrich (2007) Metrónomo de la vida cotidiana. Prácticas del tiempo de la ciudad en Europa. Gijón: Ediciones Trea.

Mückenberger, Ulrich (2008) "Familia, política de tiempo y desarrollo urbano: el ejemplo de Bremen. Revista Papers, 49: 84-93.

Mückenberger, Ulrich (2007) "Local time politices in Europe". Time and Society, 20(2): 241-273.

Murillo, Soledad (1996) El mito de la vida privada. De la entrega al tiempo propio. Madrid: Siglo XXI.

Pateman, Carole (1988) El contrato sexual. Barcelona: Editorial Anthropos [1995].

Pennachi, Laura (1990) "El símbolo dividido" Mientras tanto, 42: 53-57.

Pérez Orozco, Amaia (2006) "Atención, zona en obras: construyendo cuidadanía". Número especial conjunto sobre derechos sociales de El Ecologista, La letra A y Libre Pensamiento. Disponible en: http://www.letra.org/spip/spip.php?article4599. Consultado el 22/04/2012.

Pérez Orozco, Amaia (2010) "Diagnóstico de la crisis y respuestas desde la economía feminista". Revista de Economía Crítica, 9: 131-144.

Pérez Orozco, Amaia y Del Río, Sira (2002) La economía desde el feminismo, trabajos y cuidados. Ecologistas en Acción. Disponible en: http://www.ecologistasenaccion.org/article13104.html. Consultado el 30/05/2012.

Prieto, Carlos (2000) "Trabajo y orden social: de la nada a la sociedad de empleo (y su crisis)". Política y Sociedad, 34: 19-32.

Prieto, Carlos (2007) "Del estudio del empleo como norma social al de la sociedad como orden social". Papeles del CEIC, 1 (28): 1-28. Disponible en: http/www.ehu.es/CEIC/pdf/28.pdf. Consultado el 19/06/2011.

Ramos, Ramón (2005) "Discursos sociales del tiempo" en Guadalupe Valencia (ed.) Tiempo y Espacio. Miradas múltiples. México: CEIICH-UNAM/Plaza y Valdés, 525-543.

Ramos, Ramón (2007) "Metáforas sociales del tiempo en España: una investigación empírica" en Carlos Prieto (ed.) Trabajo, género y tiempo social. Madrid: Complutense y Hacer: 173-204.

Ramos, Ramón (2009) "Metáforas del tiempo en la vida cotidiana: una aproximación sociológica” Acta Sociológica, 49: 51-69. 
Ramos, Ramón (2011) "Más allá de las cifras: la dimensión teórica y cualitativa del tiempo" en María Ángeles Durán (dir.) El trabajo de cuidado en América y España. Madrid: Fundación Carolina-CeALCI, 75-87.

Recio, Carolina (2009) "Algunas cuestiones sobre el tiempo en la vida de las personas: tiempo y bienestar" en Carolina Recio, Elvira Méndez y Josefina Altés, Los bancos de tiempo. Experiencias de intercambio no monetario. Barcelona: Graó, 13-60.

Recio, Carolina; Méndez, Elvira y Altés, Josefina (2009) Los bancos de tiempo. Experiencias de intercambio no monetario. Barcelona: Graó.

Romañach, Javier y Lobato, Manuel (2007) "Diversidad funcional" en Luis Álvarez Pousa, et. al. Comunicación y discapacidades: actas do Foro Internacional. Colexio Profesional de Xornalistas de Galicia, Observatorio Galego dos Medios.

Sección Femenina Nacional del PCl (1990) "El tiempo: las razones de una elección" Mientras tanto, 42: 45-51.

Thompson, Edward P. (1979) "Tiempo, disciplina de trabajo y capitalismo industrial", en Tradición, revuelta y conciencia de clase. Barcelona: Crítica.

Torns, Teresa (2001) “¿Para qué un banco de tiempo?”. Mientras Tanto, 82: 117-125.

Torns, Teresa (2004) "Las políticas de tiempo: un reto para las políticas del Estado de Bienestar". Revista Andaluza de Relaciones Laborales, 13: 145-164.

Torns, Teresa (2005) "De la imposible conciliación a los permanentes malos arreglos". Cuadernos de Relaciones Laborales, 25 (1): 15-33.

Torns, Teresa (2007) "El cuidado de la dependencia. Un trabajo de cuidado". Mientras Tanto, 13: 33-43.

Torns, Teresa; Borrás, Vicent; Moreno, Sara y Recio, Carolina (2008) "Las políticas de tiempo en Europa". Revista Papers, 49: 77-84.

Protocolo para citar este texto: Legarreta Iza, M., Año, "Cuidados y sostenibilidad de la vida: Una reflexión a partir de las políticas del tiempo", en Papeles del CEIC, vol. 2014/1, $n^{\circ}$ 104, CEIC (Centro de Estudios sobre la Identidad Colectiva), Universidad del País Vasco, http://www.identidadcolectiva.es/pdf/104.pdf 
Fecha de recepción del texto: Noviembre de 2013

Fecha de evaluación del texto: Diciembre de 2013

Fecha de publicación del texto: Marzo de 2014 